\title{
Genetic variation in the NOS1AP gene is associated with the incidence of diabetes mellitus in users of calcium channel blockers
}

\author{
M. L. Becker • L. E. Visser • C. Newton-Cheh • \\ J. C. M. Witteman • A. Hofman - A. G. Uitterlinden • \\ B. H. Ch. Stricker
}

Received: 1 July 2008 /Accepted: 7 August 2008 / Published online: 3 September 2008

(C) Springer-Verlag 2008

Keywords Calcium channel blocker.

Diabetes mellitus type $2 \cdot$ Nitric oxide synthase .

Pharmacogenetics

\author{
Abbreviations \\ CCB Calcium channel blocker \\ nNOS neuronal nitric oxide synthase \\ SNP single nucleotide polymorphism
}

\footnotetext{
M. L. Becker - L. E. Visser · J. C. M. Witteman - A. Hofman •

A. G. Uitterlinden • B. H. C. Stricker $(\bowtie)$

Department of Epidemiology \& Biostatistics, Erasmus MC, P.O. Box 2040, 3000 CA Rotterdam, the Netherlands

e-mail: b.stricker@erasmusmc.nl
}

M. L. Becker - L. E. Visser

Hospital Pharmacy, Erasmus MC,

Rotterdam, the Netherlands

C. Newton-Cheh

Cardiology Division, Massachusetts General Hospital,

Boston, MA, USA

C. Newton-Cheh

Program in Medical and Population Genetics,

Broad Institute of Harvard and Massachusetts Institute

of Technology,

Cambridge, MA, USA

A. G. Uitterlinden • B. H. C. Stricker

Department of Internal Medicine, Erasmus MC,

Rotterdam, the Netherlands

\section{B. H. C. Stricker}

Drug Safety Unit, Inspectorate for Health Care,

The Hague, the Netherlands
To the Editor: Nitric oxide (NO) is an important regulator of a number of intracellular processes, including the secretion of insulin by the pancreatic beta cell. NO, produced by neuronal nitric oxide synthases (nNOS), is essential for normal beta cell functioning, and it has also been suggested that nNOS might have a role in the pathogenesis of diabetes mellitus [1-3].

Recently, the single nucleotide polymorphism (SNP) rs10494366 in the nitric oxide synthase 1 adaptor protein gene (NOS1AP), a regulator of nNOS, has been associated with QTc prolongation $[4,5]$. nNOS regulates calcium handling in the heart through the voltage-dependent calcium channels [6]. In the beta cell, exocytosis of insulin granules is triggered by an influx of calcium through these calcium channels [7]. Calcium channel blockers (CCBs) bind to a receptor on the voltage-dependent calcium channels and they regulate calcium handling by reducing the influx of calcium into the cell. As calcium channels are also situated in the pancreatic beta cell, CCBs could affect insulin secretion.

In view of these similarities in calcium handling between the myocyte and the pancreatic beta cell, we hypothesised that the SNP rs10494366 in the NOS1AP gene is associated with the incidence of diabetes mellitus in patients using CCBs. We studied this in the Rotterdam Study, a prospective population-based closed cohort study in the suburb Ommoord in Rotterdam, in which 7,983 inhabitants participated [8]. Patients were followed from 1991 onwards. Clinical examinations were carried out every 4 to 5 years and participants were monitored through linkage with files from general practitioners and pharmacies. Diabetes mellitus was diagnosed according to the World Health Organization criteria. All participants in 
the Rotterdam Study gave written informed consent. Ethical approval was obtained from the Medical Ethical Committee of the Erasmus MC.

All participants in the Rotterdam Study who received a first prescription for a CCB between 1 July 1991 and 1 January 2008 were included in the study cohort at the time of the first prescription. We excluded participants with a prescription for a CCB between 1 January 1991 and 1 July 1991 in order to have a complete medication history at the time of the first prescription and to ascertain that use of CCB after 1 July was really incident. We also excluded all participants who stopped using CCBs within 1 year, because diabetes mellitus is a disease that often has a long latent period and we assumed that duration of exposure of less than 1 year would be too short to show actual effect modification. Participants with diabetes mellitus at the first prescription for a CCB were excluded from the study cohort. Participants were followed until 90 days after the end of the last prescription for a $\mathrm{CCB}$, death or end of the study period, whichever came first.

Associations between the SNP rs10494366 in the NOS1AP gene, associated with QTc prolongation, and incidence of diabetes mellitus in current users of CCB were assessed. We adjusted for age, sex and body mass index. We developed Cox-proportional hazard models and Kaplan-Meier curves with SPSS software (version 11.0.1; SPSS Chicago, IL, USA).

In the Rotterdam Study, we identified 816 incident CCB users who were genotyped for rs10494366. The average age was 68.3 years and 342 participants were men $(41.9 \%)$. Five-hundred and forty-six participants (67\%) started with dihydropyiridine CCBs, 77 participants (9\%) with verapamil and 193 participants (24\%) with diltiazem. The minor allele frequency for rs10494366 was 0.35 (G allele).

In the study cohort, 55 participants developed diabetes mellitus while using CCBs. CCB users with the TG or GG genotype had a lower risk of incident diabetes mellitus than CCB users with the TT genotype (HR 0.56, 95\% CI 0.33-0.97). The HR in CCB users with the TG genotype (HR $0.59,95 \%$ CI $0.33-1.04$ ) was similar to the HR of CCB users with the GG genotype (HR 0.49, 95\% CI 0.19-1.26), suggesting a dominant effect of the $\mathrm{G}$ allele.

In the whole Rotterdam Study of 6,292 genotyped participants, irrespective of CCB use, no associations were found between the SNP rs10494366 and the incidence of diabetes mellitus (TG or GG genotype vs TT; HR 0.97, 95\% CI 0.83-1.14), nor was there an association in participants who were not prescribed CCBs during followup (HR 1.07, 95\% CI 0.86-1.32).

The difference in prevalence of diabetes mellitus over time between CCB users with the TT genotype and CCB

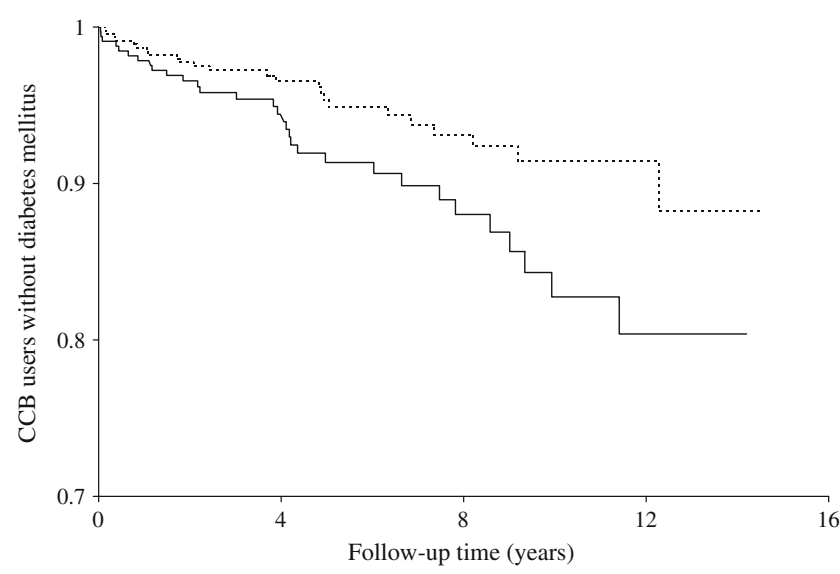

Fig. 1 Kaplan-Meier curve of differences in diabetes mellitus-free CCB users between NOS1AP genotypes. Continuous line, TT genotype; dashed line, TG or GG genotype. Logrank test, $p=0.024$. Number of participants at risk $(n)$ at $0,4,8$ and 12 years of follow-up, respectively: TT 337, 194, 92, 26; TG/GG 479, 269, 130, 32

users with the TG or GG genotype is presented in Fig. 1. During the first years of CCB therapy, the HRs of incident diabetes mellitus were similar between the genotypes. Over time the curves diverged and differences between the genotypes became larger. After 6 years of CCB therapy, the HR for $\mathrm{CCB}$ users with the TG or GG genotype compared with $\mathrm{CCB}$ users with the TT genotype was 0.42 (95\% CI 0.15-1.21). Diabetes mellitus is a progressive disease with deteriorating beta cell functioning. CCB seems to affect the progression of this deterioration differently between the rs10494366 genotypes.

In the Rotterdam Study, the total follow-up time of the participants genotyped for rs10494366 during the study period was 79,000 person-years and the total time of CCB use was 5,000 person-years. In spite of these large numbers, we only identified 55 incident diabetes mellitus patients while they were using CCBs. Our results could be a false positive result given the small number of incident cases. Therefore, to confirm this association, a large cohort in which both genotype data and drug dispensing data are available is necessary. Nevertheless, it has been demonstrated that the SNP rs10494366 is probably functional and affects calcium handling in the myocyte. Calcium handling plays a major role in insulin secretion. If replicated, our results may give a new perspective on the pathogenesis of diabetes mellitus and on the relationship between $\mathrm{CCB}$ use and the risk of developing diabetes mellitus.

Duality of interest The authors declare that there is no duality of interest associated with this manuscript. 


\section{References}

1. Lajoix AD, Reggio H, Chardes T et al (2001) A neuronal isoform of nitric oxide synthase expressed in pancreatic beta-cells controls insulin secretion. Diabetes 50:1311-1323

2. Lajoix AD, Pugniere M, Roquet F et al (2004) Changes in the dimeric state of neuronal nitric oxide synthase affect the kinetics of secretagogue-induced insulin response. Diabetes 53:1467-1474

3. Shankar RR, Wu Y, Shen HQ, Zhu JS, Baron AD (2000) Mice with gene disruption of both endothelial and neuronal nitric oxide synthase exhibit insulin resistance. Diabetes 49:684-687
4. Arking DE, Pfeufer A, Post W et al (2006) A common genetic variant in the NOS1 regulator NOS1AP modulates cardiac repolarization. Nat Genet 38:644-651

5. Aarnoudse AJ, Newton-Cheh C, de Bakker PI et al (2007) Common NOS1AP variants are associated with a prolonged QTc interval in the Rotterdam Study. Circulation 116:10-16

6. Schulz R, Rassaf T, Massion PB, Kelm M, Balligand JL (2005) Recent advances in the understanding of the role of nitric oxide in cardiovascular homeostasis. Pharmacol Ther 108:225-256

7. Ashcroft FM, Rorsman P (1989) Electrophysiology of the pancreatic beta-cell. Prog Biophys Mol Biol 54:87-143

8. Hofman A, Breteler MM, van Duijn CM et al (2007) The Rotterdam Study: objectives and design update. Eur J Epidemiol 22:819-829 